\title{
Applicability of common reference intervals for serum creatinine concentrations to the Croatian population
}

\author{
Zlata Flegar-Meštrićc ${ }^{1, *}$, Sonja Perkov ${ }^{1}$ Barbara \\ Šimonović $^{1}$ and Dubravka Juretić ${ }^{2}$ \\ ${ }^{1}$ Institute of Clinical Chemistry "'Merkur', University \\ Hospital, Zagreb, Croatia \\ ${ }^{2}$ Croatian Society of Medical Biochemists, Zagreb, Croatia
}

\begin{abstract}
Background: In accordance with an ongoing activity for worldwide harmonization based on traceability in laboratory methods, the goal of this study was to validate the applicability of recommended "common" reference intervals for serum creatinine concentrations using a specific enzymatic method to the Croatian population.

Methods: The reference group consisted of 240 healthy subjects (120 males and 120 females), between 18 and 74 years of age (median 57 years), who were selected in accordance with the International Federation of Clinical Chemistry and Laboratory Medicine (IFCC) recommendations. Creatinine in serum was measured using the creatinine enzymatic assay (Olympus OSR61204) that was standardized to the isotopic dilution mass spectrometry (IDMS) method and National Institute of Standards and Technology (NIST) Standard Reference Material (SRM) 967. In addition, creatinine was measured using a kinetic Jaffe method (Olympus OSR6178) standardized to NIST SRM 909b level 2 standard.
\end{abstract}

Results: Method comparison between enzymatic creatinine (x) and the Jaffe kinetic method (y) gave the following P/B equation for the entire group $(n=240)$ : $y=1.00 x+17.00$; $r=0.968$. Reference intervals for serum creatinine (central 95th percentiles) obtained using the enzymatic creatinine method ranged from 54 to $107 \mu \mathrm{mol} / \mathrm{L}$ for males and from 50 to $93 \mu \mathrm{mol} / \mathrm{L}$ for females. The IFCC recommended common reference intervals for global applications are 64-104 $\mu \mathrm{mol} / \mathrm{L}$ and $49-90 \mu \mathrm{mol} / \mathrm{L}$ for males and females, respectively.

Conclusions: Comparability of obtained results confirmed the applicability of recently recommended "common" reference intervals to the Croatian population for all laboratories measuring serum creatinine concentrations using enzymatic methods traceable to the IDMS method and NIST SRM 967. Clin Chem Lab Med 2010;48:231-5.

\footnotetext{
*Corresponding author: Zlata Flegar-Meštrić, Institute of Clinical Chemistry 'Merkur'” University Hospital, Zajčeva 19, 10000 Zagreb, Croatia

Phone/Fax: +38512431397,

E-mail: zlata.mestric@ zg.t-com.hr

Received September 8, 2009; accepted October 19, 2009;

previously published online November 30, 2009
}

Keywords: applicability of reference intervals; calibration traceability; creatinine enzymatic method; serum creatinine concentration.

\section{Introduction}

Variability of the clinically relevant blood constituents throughout an individual's lifetime depends on various physiological processes and numerous biological factors which define characteristics of any population $(1,2)$. Population based reference intervals for clinically relevant blood and serum constituents for the Croatian population were produced under strictly controlled conditions according to the International Federation of Clinical Chemistry and Laboratory Medicine (IFCC) recommendations proposed by the Clinical and Laboratory Standards Institute (CLSI) C28 document (3). These reference intervals were based upon a representative reference sample group of 2246 adults and 998 children, aged 8-70 years, during the period from 1998 to 2000 (4-7). All tests were performed by the Institute of Clinical Chemistry at the University Hospital Merkur, "Reference Center of Ministry of Health and Social Care for Producing Reference Values in the Field of General Medical Biochemistry', Zagreb, Croatia, using routine analytical methods. Serum creatinine concentrations were measured using an uncompensated kinetic Jaffe method. In order to harmonize laboratory results, the Croatian Chamber of Medical Biochemists recommended the application of obtained reference intervals for all laboratories using the same analytical methods in January, 2005 (8). Results that are obtained are evaluated through the laboratories participation in the national External Quality Assessment (EQA). The EQA has been performed continuously since 1973 by the Committee for External Quality Assessment in Medical Biochemical Laboratories under the auspices of the Croatian Society of Medical Biochemists (9). A review of long-term results collected from EQA surveys has shown that serum creatinine concentration in almost 200 medical biochemical laboratories in Croatia are measured with average $\mathrm{CV}$ between $4.3 \%$ and $6.2 \%$ when using an uncompensated kinetic Jaffe method. National results for creatinine concentrations in the International Measurement Evaluation Program-17 (IMEP-17) measured with Jaffe methods in 28 medical biochemical laboratories in clinical hospitals showed a significantly positive bias to the isotopic dilution mass spectrometry (IDMS) certified reference material (CRM), with differences up to $30 \%$ (10).

Due to the recent international requirement for accurate and specific diagnostic tests and implementation of calibra- 
tion traceability to high order reference measurement procedures and reference materials (11-14), the goal of this study was to validate the applicability of recommended "common" reference intervals for serum creatinine concentrations using a specific enzymatic method. We wished to validate reference intervals in the Croatian population using the enzymatic method instead of previously produced reference intervals established using an uncompensated kinetic Jaffe creatinine method. This work was performed as a prerequisite for standardization of serum creatinine measurements and harmonization of measured results.

\section{Materials and methods}

\section{Reference individuals and samples}

The reference population group consisted of 240 healthy adults (120 males and 120 females), aged between 18 and 74 years (median 57 years), from an urban population of Zagreb, Croatia. During 2008, all subjects were selected and declared healthy on the basis of a questionnaire and results of standard laboratory tests including measurement of alanine and aspartate aminotransferase, alkaline phosphatase, $\gamma$-glutamyltransferase, cholinesterase, lactate dehydrogenase, glucose, total proteins, total bilirubin, urea, urate, C-reactive protein and a complete blood count. Before collection of blood, each individual completed a simple questionnaire requesting information about age, gender, height, weight, heredity for diabetes, severe disease, prescribed drugs, food preferences and cigarette smoking. None of reference individuals had a history of severe diseases and none of the women were pregnant. Smokers were included while individuals taking any prescribed drugs were excluded from the reference population group. None of the reference individuals were vegetarian. Women had a mean weight of $71.5 \mathrm{~kg}(\mathrm{~s}=8.9 \mathrm{~kg})$ and a mean height of $1.64 \mathrm{~m}(\mathrm{~s}=0.05 \mathrm{~m})$ and men had a mean weight of $86.1 \mathrm{~kg}(\mathrm{~s}=10.6 \mathrm{~kg})$ and a mean height of $1.87 \mathrm{~m}(\mathrm{~s}=0.05 \mathrm{~m})$. Results of standard laboratory tests for all subjects were within previously established reference intervals for the Croatian population $(4,5,7)$. This study was performed with permission from the Ethical Committee of Medical School University of Zagreb, Croatia, and written consent was obtained from all participants.

Venous blood was collected under controlled conditions into vacutainer tubes (Becton Dickinson, Plymouth, England) containing no additives. Serum was prepared $30 \mathrm{~min}$ after blood collection by centrifugation at $1800 \times g$, and sera were frozen at $-80^{\circ} \mathrm{C}$ until analysis.

\section{Measurement methods}

All measurements were performed using an Olympus AU 600 analyzer (Olympus Mishima Co., Ltd., Shizuoka, Japan) using Olympus reagents. Serum creatinine concentrations were measured using the conventional kinetic uncompensated Jaffe method (Olympus OSR6178) and by an enzymatic assay (Olympus OSR61204). The analyzer was calibrated using the manufacturer's calibrator (Olympus Cat. No. 66300). The creatinine concentration for the serum calibrator was traceable to the IDMS method and National Institute of Standards and Technology (NIST) Standard Reference Material (SRM) 967 for the enzymatic method, and to the NIST SRM 909b level 2 for the conventional kinetic uncompensated Jaffe method. The commercial control sera for normal (ODC0003 Olympus control level 1) and pathological (ODC0004 Olympus control level 2) concentrations were used for evaluation of metrological characteristics of the measurement procedures. Reference intervals were calculated using MedCalc statistical software (http://www.medcalc.be). The established reference intervals reflected our estimate of the 2.5-97.5 percentiles for a non-parametric distribution.

Regression analysis for comparison of methods was done using the non-parametric Passing/Bablok method, and a linear regression model was used to establish correlation coefficients.

\section{Results}

\section{Quality control data}

During the study, the same control materials were used, the Olympus control serum level 1 and Olympus control serum level 2, Ireland. Metrological characteristics of measurement procedures used to produce reference values for creatinine concentrations in serum are shown as day-to-day imprecision and the relative systematic error. Relative systematic errors were calculated with respect to the assigned values of control materials. The data for long-term analytical imprecision and inaccuracy (expressed as the relative systematic error) obtained under controlled pre-analytical conditions are presented in Table 1. Our results showed better metrological characteristics for the enzymatic creatinine method calibrated to NIST SRM 967 as compared to the uncompensated Jaffe method calibrated to NIST SRM 909b level 2. Based on biological variability, current desired goals for laboratory performance for creatinine measurements are $2.8 \%$ for inaccuracy and $2.2 \%$ for imprecision (15). Considering both imprecision and inaccuracy in making an estimate of

Table 1 Metrological characteristics for serum creatinine measurements using the uncompensated kinetic Jaffe method and enzymatic method with two concentrations of commercially available controls.

\begin{tabular}{|c|c|c|c|c|c|c|c|c|c|}
\hline \multirow[t]{3}{*}{ Quantity } & \multirow[t]{3}{*}{ Method } & \multicolumn{8}{|c|}{ Metrological quality } \\
\hline & & \multicolumn{4}{|c|}{ Olympus control serum 1} & \multicolumn{4}{|c|}{ Olympus control serum 2} \\
\hline & & $\mathrm{n}$ & $\mathrm{x}$ & $\mathrm{CV}_{\mathrm{M}}$ & $\mathrm{e}_{\mathrm{Sr}}$ & $\mathrm{n}$ & $\mathrm{x}$ & $\mathrm{CV}_{\mathrm{M}}$ & $\mathrm{e}_{\mathrm{Sr}}$ \\
\hline \multirow{2}{*}{$\begin{array}{l}\text { S-creatinine; } \\
\text { subst.c., } \mu \mathrm{mol} / \mathrm{L}\end{array}$} & Jaffe kinetic & 71 & 116.3 & 2.7 & 0.3 & 71 & 455.3 & 2.3 & 0.7 \\
\hline & Enzymatic & 48 & 107.4 & 2.3 & 0.6 & 79 & 454.1 & 1.5 & 1.1 \\
\hline
\end{tabular}

$\mathrm{S}$, serum; subst.c., substance concentration; $\mathrm{n}$, number of control results; $\mathrm{x}$, mean concentration observed; $\mathrm{CV}_{\mathrm{M}}$, day-to-day imprecision $(\%) ; \mathrm{e}_{\mathrm{Sr}}$, relative systematic error (\% with regard to the assigned value). 
analytical performance required to meet the clinical interpretation goals for serum creatinine measurements, the recommended desirable goal of $8.2 \%$ for total error (16) was achieved for both methods.

\section{Method comparison}

Method comparison between the enzymatic creatinine method (x) and uncompensated Jaffe kinetic method (y) gave the following Passing/Bablok equation for the whole group of 240 individuals: $y=1.00 x+17.00$ and a correlation coefficient of 0.968. As expected, the creatinine uncompensated Jaffe method gave substantially higher values compared with the enzymatic method. These results are in accordance with several studies that compared an enzymatic method with the non-specific conventional Jaffe method. These results indicate that Jaffe methods, based on an alkaline picrate reaction, overestimate true serum creatinine concentrations due primarily to non-specific protein interference (17-19).

\section{Reference intervals for serum creatinine}

The central 95th percentile reference interval was determined using the reference population of 240 healthy adult persons. The interval obtained using the enzymatic assay traceable to IDMS and SRM 967 ranged from 54 to $107 \mu \mathrm{mol} / \mathrm{L}$ in males, and from 50 to $93 \mu \mathrm{mol} / \mathrm{L}$ in females. These ranges can be compared to the recommended common reference intervals for global application of 64-104 and 49-90 $\mu \mathrm{mol} / \mathrm{L}$ for males and females, respectively (Table 2).

The reference intervals for the uncompensated kinetic Jaffe method traceable to the IDMS method and SRM 909b level 2 was found to be $70-126 \mu \mathrm{mol} / \mathrm{L}$ in males and $66-110 \mu \mathrm{mol} / \mathrm{L}$ in females. This range was almost identical with the reference intervals produced in our previous study (6) using a group of 2246 healthy adults: 79-125 $\mu \mathrm{mol} / \mathrm{L}$ and 63-107 $\mu \mathrm{mol} / \mathrm{L}$ for males and females, respectively. Detailed data are presented in Table 3.

\section{Discussion}

Reference intervals for healthy subjects are very important for the clinical interpretation of laboratory test values (21, 22). The quality of reference intervals can play a large role in result interpretation as the quality of the result itself. The International Organization for Standardization (ISO) and Standard 15189 requests that "biological reference intervals shall be periodically reviewed"' (23). The European Union Directive on In Vitro Diagnostics Medical Devices (IVD MD) asks manufacturers to provide detailed information on reference intervals and to provide traceability to reference methods and materials for analytes (13). More recently, the NIST prepared a secondary commutable creatinine reference material designated SRM 967 which became available to the IVD industry (24). SRM 967 has a frozen serum matrix and is intended for use by laboratories and in vitro diagnostics equipment manufactures for the calibration and evaluation of
Table 2 Common reference intervals for creatinine concentrations in serum compared to reference intervals for adult Croatian population produced using an enzymatic method traceable to the IDMS method and NIST SRM 967.

\begin{tabular}{llr}
\hline Age (gender) group & \multicolumn{2}{l}{ Percentile value, $\mu \mathrm{mol} / \mathrm{L}$} \\
\cline { 2 - 3 } & 2.5 th & 97.5 th \\
\hline Common reference intervals & & \\
for global application (20) & & 86 \\
Cord sera & 46 & 81 \\
Term neonates 0-14 d & 27 & 34 \\
2 m-<1 y & 14 & 31 \\
1 y-<3 y & 15 & 37 \\
3 y-<5 y & 23 & 42 \\
5 y-<7 y & 25 & 48 \\
7 y-<9 y & 30 & 57 \\
9 y-<11 y & 28 & 63 \\
11 y-<13 y & 37 & 72 \\
13 y-<15 y & 40 & 104 \\
Adult (males) & 64 & 90 \\
Adult (females) & 49 & \\
Reference intervals in the & & \\
reference sample group of & & \\
Croatian population ( $=240)$ & & \\
Adult (males) & 54 & 507 \\
Adult (females) & 50 & \\
\hline
\end{tabular}

d, days; m, months; y, years; n, number of reference subjects.

routine clinical methods (25). Several studies indicate that introducing specific enzymatic methods for creatinine measurements in the routine laboratory should guarantee traceability of each individual reference result to the reference measurement system $(17,20)$. Because the NIST SRM 909b

Table 3 Reference intervals for creatinine concentrations in serum for school children and adolescents $(n=998)$ and adults $(n=2246)$ in Croatians compared to current data obtained in the reference sample group of adult reference individuals from the same population using conventional uncompensated Jaffe kinetic method traceable to the IDMS method and NIST SRM 909b level 2.

\begin{tabular}{llr}
\hline Age (gender) group & \multicolumn{2}{c}{ Percentile value, $\mu \mathrm{mol} / \mathrm{L}$} \\
\cline { 2 - 3 } & 2.5 th & 97.5 th \\
\hline Reference intervals in & & \\
Croatians $(4,6)$ & & 80 \\
8 y $-<13$ y (males, females) & 46 & 104 \\
14 y- $<18$ y (males) & 60 & 96 \\
14 y-<18 y (females) & 57 & 125 \\
20 y-<70 y (males) & 79 & 107 \\
20 y-<70 y (females) & 63 & \\
Current data on reference & & \\
intervals in the reference & & 126 \\
sample group of Croatians & & \\
(n=240) & & 110 \\
Adult (males) & 70 & \\
Adult (females) & 66 & \\
\hline
\end{tabular}

$\mathrm{y}$, years; $\mathrm{n}$, number of reference subjects. 
reference material that was previously used is not commutable with native human serum, it is unsuitable for direct calibration of routine methods (24).

In order to establish traceability for creatinine measurements and to harmonize laboratory results and their interpretation for clinical use, we validated the applicability of recommended "common" reference intervals for serum creatinine concentrations using an enzymatic method traceable to the IDMS method and SRM 967 to the Croatian population. This was done instead of using reference intervals established previously using an uncompensated kinetic Jaffe method traceable to the IDMS method and SRM 909b level 2. Creatinine results for the reference population group of 240 healthy adult persons obtained under controlled pre-analytical conditions and with analytical quality within desirable target values using a specific enzymatic creatinine method showed essentially identical results compared with recently recommended "common" reference intervals for global application by the IFCC Committee on Reference Intervals and Decision Limits (20). Due to the fact that creatinine concentrations with respect to age and gender in our populationbased study measured using an uncompensated kinetic Jaffe method (4-6) are quite similar to those reported in recently recommended "common" reference intervals for global application (20), it appears that "common" reference intervals could be adopted for all age groups. Based on validation results, we obtained, all prerequisites for adopting established common reference intervals for creatinine measurement were fulfilled.

Recommended "common', reference intervals for global application could be used for creatinine measurement on a national level by all laboratories that use a standardized, specific enzymatic method traceable to the commutable highorder reference material NIST SRM 967. In addition, they need to demonstrate that using controlled pre-analytical conditions, their analytical performance meets the recommended performance goal of $<10 \%$ total error based on biological variability and clinical requirements $(15,26,27)$. According to guidelines from the CLSI, validation of reference intervals can be performed using a small sample of 20 reference individuals from the laboratory's population (28).

In case of serum creatinine, standardization is of particular importance because of its role in the assessment of renal function and for estimation of glomerular filtration rate (29, 30). Introducing the enzymatic creatinine method to routine laboratory work, instead of the alkaline picrate method, is in accordance with recent recommendations of the Laboratory Working Group of the National Kidney Disease Education Program. This group suggests that the estimated glomerular filtration rate has to be reported using accurate and specific serum creatinine measurements, based on the concept of traceability (16).

\section{Conflict of interest statement}

Authors' conflict of interest disclosure: The authors stated that there are no conflicts of interest regarding the publication of this article.
Research funding: None declared.

Employment or leadership: None declared.

Honorarium: None declared.

\section{References}

1. Ceriotti F, Hinzmann R, Panteghini M. Reference intervals: the way forward. Ann Clin Biochem 2009;46:8-17.

2. Horn PS, Pesce AJ. Reference intervals: an update. Clin Chim Acta 2003;334:5-23.

3. Determination of reference intervals in the clinical laboratory using the proposed guideline National Committee for Clinical Laboratory Standards C28-P. Arch Pathol Lab Med 1992;116: $710-3$.

4. Jagarinec N, Flegar-Meštrić Z, Šurina B, Vrhovski-Hebrang D, Preden-Kereković V. Pediatric reference intervals for 34 biochemical analytes in urban school children and adolescents. Clin Chem Lab Med 1998;36:327-37.

5. Flegar-Meštrić Z, Tadej D, Vrhovski-Hebrang D. Reference intervals of 56 haematological and biochemical constituents of blood and serum for reference individuals corresponding to "reference state"'-population of Zagreb, Croatia. Croat Med J 1993;34:163-9.

6. Flegar-Meštrić Z, Preden-Kereković V, Vrhovski-Hebrang D, Šurina B, Nazor A. Reference intervals of haematological and biochemical constituents of blood and serum in the age of 8-70 years [in Croatian]. Biochemia Medica 2000;10:1-9.

7. Flegar-Meštrić Z, Nazor A, Jagarinec N. Haematological profile in healthy urban population (8-70 years of age). Coll Antropol 2000;24:185-96.

8. Flegar-Meštrić Z, Juretić D, Čvorišćec D. Harmonization of laboratory medicine in Croatia [in Croatian]. Liječ Vjesn 2006;128:183-8.

9. Juretić D, Čepelak I, Flegar-Meštrić Z, Nazor A, Parag G, Sikirica $\mathrm{M}$. External quality assessment program for medical biochemical laboratories in Croatia. Clin Chem Lab Med 2002; 40(Suppl M-RE-003):139.

10. Örnemark U, Van Nevel L, Smeyers P, Harper C, Taylor PD. The International Measurement Evaluation Program IMEP-17. Trace and minor constituents in human serum. EUR 20694 EN. Report to participants. Part 2: methodology and quantity specifications. www.imep.ws. Accessed August 1, 2009.

11. Vesper HW, Thienpont LM. Traceability in laboratory medicine. Clin Chem 2009;55:1067-75.

12. Klee GG. Assay configuration and analytic specificity may have major effects on prediction of clinical outcomes - implications for reference standards. Clin Chem 2009;55:848-9.

13. Directive 98/79/EC of the European Parliament and of the Council of 27 October 1998 on in vitro diagnostic medical devices. Off J Eur Communities 1998:L331/1-37.

14. Thienpont LM, Van Uytfanghe K, Rodríguez Cabaleiro D. Metrological traceability of calibration in the estimation and use of common medical decision-making criteria. Clin Chem Lab Med 2004;42:842-50.

15. Ricos C, Alvarez V, Cava F, Garcia-Lario JV, Hernandez A, Jimenez CV, et al. Current databases on biological variation: pros, cons and progress. Scand J Clin Lab Invest 1999;59:491500. The 2008 update. http://www.westgard.com/guest26.htm.

16. Myers GL, Miller WG, Coresh J, Fleming J, Greenberg N, Greene $\mathrm{T}$, et al. Recommendations for improving serum creatinine measurement: a report from the Laboratory Working 
Group of the National Kidney Disease Education Program. Clin Chem 2006;52:5-18.

17. Panteghini M on behalf of the IFCC Scientific Division. Enzymatic assays for creatinine: time for action. Clin Chem Lab Med 2008;46:567-72.

18. Delanghe JR, Cobbaert C, Galteau MM, Harmoinen A, Jansen $\mathrm{R}$, Kruse $\mathrm{R}$, et al. Trueness verification of actual creatinine assays in the European market demonstrates a disappointing variability that needs substantial improvement. Clin Chem Lab Med 2008;46:1319-25.

19. Chromý V, Rozkošná K, Sedlák P. Determination of serum creatinine by Jaffe method and how to calibrate to eliminate matrix interference problems. Clin Chem Lab Med 2008;46: 1127-33.

20. Ceriotti F, Boyd JC, Klein G, Henny J, Queraltó J, Kairisto V, et al. Reference intervals for serum creatinine concentrations: assessment of available data for global application. Clin Chem 2008;54:559-66.

21. Gräsbeck R. The evolution of the reference value concept. Clin Chem Lab Med 2004;42:692-7.

22. Fraser CG. Inherent biological variation and reference values. Clin Chem Lab Med 2004;42:758-64.

23. Croatian Standard: HRN EN ISO 15189:2008 Medical laboratories - particular requirements for quality and competencies (ISO 15189:2007; EN ISO 15189:2007). ISO, Geneva, Switzerland.
24. Panteghini M, Myers GL, Miller WG, Greenberg N. IFCC. The importance of metrological traceability on the validity of creatinine measurement as an index of renal function. Clin Chem Lab Med 2006;44:1287-92.

25. Dodder NG, Tai SS-C, Sniegoski T, Zhang NF, Welch MJ. Certification of creatinine in a human serum reference material by GC-MS and LC-MS. Clin Chem 2007;53:1694-9.

26. Peake M, Whiting M. Measurement of serum creatinine - current status and future goals. Clin Biochem Rev 2006;27:17384.

27. Jones G, Barker A. Reference intervals. Clin Biochem Rev 2008;29(Suppl i):93-7.

28. Clinical and Laboratory Standards Institute. How to define and determine reference intervals in the clinical laboratory: approved guideline, 2nd ed. CLSI document C28-A2. Wayne, PA, USA: CLSI, 2000.

29. Fuentes-Arderiu X, Álvarez-Funes V, Coca-Fábregas L, CruzPlacer M, Díaz-Fernández J, Herrero-Bernal P, et al. Multicentre physiological reference values for the concentration of creatininium in plasma and diagnostic specificity of glomerular filtration rate estimated with the MDRD equation. Clin Chem Lab Med 2007;45:531-4.

30. Van Biesen W, Vanholder R, Veys N, Verbeke F, Delanghe J, De Bacquer D, et al. The importance of standardization of creatinine in the implementation of guidelines and recommendations for CKD: implications for CKD management programmes. Nephrol Dial Transplant 2006;21:77-83. 